\title{
Consistency and Linearity in Quantum Theory
}

\author{
Ariel Caticha \\ Department of Physics, University at Albany-SUNY, Albany, NY 12222
}

\begin{abstract}
Quantum theory is formulated as the uniquely consistent way to manipulate probability amplitudes. The crucial ingredient is a consistency constraint: if the amplitude of a quantum process can be computed in two different ways, the two answers must agree. The constraint is expressed in the form of functional equations the solution of which leads to the usual sum and product rules for amplitudes. An immediate consequence is that the Schrödinger equation must be linear: non-linear variants of quantum mechanics violate the requirement of consistency.

PACS: 03.65.Bz, 03.65.Ca.
\end{abstract}

The formalism of quantum mechanics is very robust; modifications are invariably unsuccessful. A number of non-linear alternative theories have been proposed, 1 experimentally tested, 21 and promptly discarded, but the question of whether new non-linear variants might prove successful is not settled. In fact, interest in the subject remains in spite of, or perhaps, partly because of the possibility that these theories might allow super-luminal communication. 3 .

In this paper we propose an explanation for the robustness of quantum mechanics. The basic argument is analogous to one used by R. T. Cox in the very different context of classical probability theory. He showed that once degrees of probability are represented by real numbers there is a unique set of rules for reasoning under conditions of insufficient information. not only legitimized the view of probability theory as an extended form of logic, but also provided an explanation for its uniqueness, for its inevitability.

The crux of our argument is (like Cox's) a consistency requirement: if the amplitude (i.e., the probability amplitude) for a quantum process can be computed in two different ways, the two answers must agree. This requirement is expressed in the form of functional equations, the solution of which leads to the usual sum and product rules for amplitudes. 6] An immediate consequence is that the equation for time evolution, the Schrödinger equation, is necessarily linear. In other words, the usual linear quantum theory emerges as the unique way to manipulate amplitudes consistently.

To emphasize the simplicity of our approach we will focus on a simple particle with no spin; its only attribute is its position. The generalization to other more complex systems should in principle be straightforward. 
The first step is pragmatic: we identify the statements we are allowed to make about the particle with the idealized experimental setups designed to test them. As will become clear below this set of allowed propositions or setups is more restricted than those used in other approaches based on either quantum logic, [1-19 or consistent histories 10 or on Bayesian complex probabilities. 11

Suppose that what we can detect is the presence or absence of a particle in a small region of space-time around an event $x=(\vec{x}, t)$. The simplest statement describing motion, "the particle moves from $x_{i}$ to $x_{f}$," will be denoted by $\left[x_{f}, x_{i}\right]$. It can be tested by preparing a particle at $x_{i}$ and placing a detector at $x_{f}$.

The more complex proposition "the particle goes from $x_{i}$ to $x_{1}$ and from there to $x_{f}$ " (we assume that $t_{i}<t_{1}<t_{f}$ ) will be denoted by $\left[x_{f}, x_{1}, x_{i}\right]$. To test it we need to introduce an idealized device, a "filter" which prevents any motion from $x_{i}$ to $x_{f}$ except via the intermediate event $x_{1}$. This filter is some sort of obstacle or screen that exists only at time $t_{1}$, blocking the particle everywhere in space except for a small "hole" around the point $\vec{x}_{1}$.

We can also open two holes, one at $\vec{x}_{1}$ and another at $\vec{x}_{1}^{\prime}$ in our filter at $t_{1}$. This will allow testing whether "the particle goes from $x_{i}$ to $x_{f}$ via point $x_{1}$ or $x_{1}^{\prime}$," which we may write as $\left[x_{f},\left(x_{1}, x_{1}^{\prime}\right), x_{i}\right]$.

The possibility of introducing many filters each with many holes leads to allowed propositions or setups of the general form

$$
a=\left[x_{f}, s_{N}, s_{N-1}, \ldots, s_{2}, s_{1}, x_{i}\right],
$$

where $s_{n}=\left(x_{n}, x_{n}^{\prime}, x_{n}^{\prime \prime}, \ldots\right)$ is a filter at time $t_{n}$, intermediate between $t_{i}$ and $t_{f}$, with holes at $\vec{x}_{n}, \vec{x}_{n}^{\prime}, \vec{x}_{n}^{\prime \prime}, \ldots$ These propositions involving a single initial and a single final event are special cases of what are usually called histories. 10,12

Our second step starts from the observation that if two setups are related in some way then information about one may be relevant to predictions about the other. A relation of this kind arises when two setups $a$ and $b$ are placed in immediate succession resulting in a third setup which will be denoted by $a b$. This operation, which we will call and, cannot be used to combine any two arbitrarily chosen propositions $a$ and $b$; it is necessary that the destination point of the earlier setup coincide with the source point of the later setup, otherwise the combined $a b$ is not an allowed proposition. For example,

$$
\left[x_{f}, s_{N}, \ldots, s_{n+1}, x_{n}\right]\left[x_{n}, s_{n-1}, \ldots, s_{1}, x_{i}\right]=\left[x_{f}, s_{N}, \ldots, x_{n}, \ldots, s_{1}, x_{i}\right] .
$$

It is crucial that all $t_{1}, \ldots, t_{n-1}$ happen before $t_{n}$ and that $t_{n+1}, \ldots, t_{N}$ happen after $t_{n}$, otherwise the two setups are not consecutive.

Another useful relation arises when two setups $a^{\prime}$ and $a^{\prime \prime}$ are identical except on one single filter where none of the holes of $a^{\prime}$ overlap any of the holes of $a^{\prime \prime}$. We may then form a third setup $a$, denoted by $a^{\prime} \vee a^{\prime \prime}$, which includes the holes of both $a^{\prime}$ and $a^{\prime \prime}$. An example of this operation, which we will call or, is

$$
\left[x_{f}, \ldots, s_{n}^{\prime}, \ldots, x_{i}\right] \vee\left[x_{f}, \ldots, s_{n}^{\prime \prime}, \ldots, x_{i}\right]=\left[x_{f}, \ldots, s_{n}, \ldots, x_{i}\right],
$$


where

$$
s_{n}^{\prime}=\left(x_{n}^{\prime 1}, x_{n}^{\prime 2}, \ldots, x_{n}^{\prime j}\right), s_{n}^{\prime \prime}=\left(x_{n}^{\prime \prime 1}, x_{n}^{\prime \prime 2}, \ldots, x_{n}^{\prime \prime k}\right),
$$

and

$$
s_{n}=\left(x_{n}^{\prime 1}, \ldots, x_{n}^{\prime j}, x_{n}^{\prime \prime 1}, \ldots, x_{n}^{\prime \prime k}\right) .
$$

Again, notice that it is only for special choices of propositions $a$ and $b$ that this or operation will result in an allowed proposition $a \vee b$.

The basic properties of and and or can be obtained from the principle that two propositions are equal when they are tested by setups with the same distribution of filters and holes. For example, the or operation is commutative, but and is not

$$
a \vee b=b \vee a, \quad a b \neq b a .
$$

There is an asymmetry implicit in the idea of placing setups in succession: one setup is the earlier one. If $a b$ is allowed, $b a$ is not. The next properties concern associativity. Given three consecutive setups $a, b$ and $c$ we can write

$$
(a b) c=a(b c) \equiv a b c,
$$

provided $a b$ and $b c$ are allowed (then $a b c$ is allowed too). Similarly, for the or operation we have

$$
(a \vee b) \vee c=a \vee(b \vee c) \equiv a \vee b \vee c,
$$

provided $(a \vee b),(b \vee c),(a \vee b) \vee c$ and $a \vee(b \vee c)$ are all allowed. Finally, and and or enjoy a measure of distributivity,

$$
a(b \vee c)=(a b) \vee(a c) \text { or }(b \vee c) a=(b a) \vee(c a)
$$

Which of these two holds, if any, depends again on whether the relevant propositions are allowed.

The quantum and and or operations introduced here are not logical but rather physical connectives; they represent our idealized ability to construct more complex physical setups out of simpler ones. They differ from their Boolean and quantum logic analogues in that they do not operate between arbitrary propositions (see the paragraphs above eqs. (2) and (3)). Furthermore, distributivity fails in quantum logics but not in the present approach.

Next, we assume that a quantitative representation of the relations (and/or) between setups can be obtained by assigning to each setup $a$ a single complex number $\phi(a)$. By a 'representation' we mean that the assignment of $\phi \mathrm{s}$ is such that relations among setups translate into relations among the corresponding complex numbers. But why should such a representation exist? Why complex 
numbers? No answer here; this is the mysterious feature of quantum theory. It seems that a single complex number is sufficient to convey the physically relevant information about a setup.

To be specific, suppose that two setups $a$ and $a^{\prime}$ can be combined into $a \vee a^{\prime}$. Then given the numbers $\phi(a)$ and $\phi\left(a^{\prime}\right)$ and the fact that the relevant relation is or one should be able to calculate $\phi\left(a \vee a^{\prime}\right)$ : there must exist a function $S$ such that

$$
\phi\left(a \vee a^{\prime}\right)=S\left(\phi(a), \phi\left(a^{\prime}\right)\right),
$$

and that this same function $S$ apply to any other setups that are similarly related. Thus, $S$ represents or.

The existence of $S$ constrains the assignment of $\phi$ s. For example, the number $\phi\left(a \vee a^{\prime} \vee a^{\prime \prime}\right)$ can be calculated either as $\phi\left(\left(a \vee a^{\prime}\right) \vee a^{\prime \prime}\right)$ or as $\phi\left(a \vee\left(a^{\prime} \vee a^{\prime \prime}\right)\right)$, and the two ways must agree,

$$
S\left(\phi\left(a \vee a^{\prime}\right), \phi\left(a^{\prime \prime}\right)\right)=S\left(\phi(a), \phi\left(a^{\prime} \vee a^{\prime \prime}\right)\right) .
$$

Further use of $S$ leads to the consistency constraint

$$
S(S(u, v), w)=S(u, S(v, w)),
$$

where we have put $\phi(a)=u, \phi\left(a^{\prime}\right)=v$, and $\phi\left(a^{\prime \prime}\right)=w$. One can check, by substitution, that eq. (10), is satisfied if

$$
S(u, v)=\xi^{-1}(\xi(u)+\xi(v)),
$$

or,

$$
\xi(S(u, v))=\xi(u)+\xi(v),
$$

where $\xi$ is an arbitrary function. Different choices of $\xi$ lead to different, equally acceptable forms of $S$. The proof by Cox that eq. (12) is the general solution in the case of real variables holds in the complex case as well. Conversely, 4,13 if the function $S$ exists then there must also exist another function $\xi$, calculable from $S$, such that

$$
\xi\left(\phi\left(a \vee a^{\prime}\right)\right)=\xi(\phi(a))+\xi\left(\phi\left(a^{\prime}\right)\right) .
$$

This result shows that, instead of the original $\phi(a)$, an equivalent and much more convenient assignment is the number $\xi(\phi(a))$, which we will denote by $\xi(a)$. In other words, the assignment of a number $\xi(a)$ to a proposition $a$ can always be done so that $o r$ is represented by a simple sum rule,

$$
\xi\left(a \vee a^{\prime}\right)=\xi(a)+\xi\left(a^{\prime}\right) .
$$

In this representation $S$ is addition.

Similarly, and is represented by a function $P$ such that

$$
\xi(a b)=P(\xi(a), \xi(b)),
$$


whenever $a, b$, and $a b$ are allowed propositions. The previous argument can be repeated: the associativity of and constrains $P$ through the requirement that $\xi(a b c)$ be calculable either as $\xi((a b) c)$ or as $\xi(a(b c))$. Therefore,

$$
P(P(u, v), w)=P(u, P(v, w)),
$$

where $\xi(a)=u, \xi(b)=v$, and $\xi(c)=w$. Our freedom to choose $P$ is further limited, and quite severely so, by our previous choice of $S$. The distributivity of and relative to or yields an additional constraint: since the number $\xi(a(b \vee c))$ can also be computed as $\xi((a b) \vee(a c))$ consistency requires that

$$
P(u, v+w)=P(u, v)+P(u, w) .
$$

Equation (15) implies that there must exist a function $\eta$, calculable from $P$, such that

$$
\eta(P(u, v))=\eta(u)+\eta(v) .
$$

Taking the exponential of both sides, and letting $\zeta=e^{\eta}$, transforms this sum into a product,

$$
\zeta(P(u, v))=\zeta(u) \zeta(v) \quad \text { or } \quad P(u, v)=\zeta^{-1}(\zeta(u) \zeta(v)) .
$$

Substitution into eq. (16), leads to a functional equation for $\zeta$,

$$
\zeta^{-1}(\zeta(u) \zeta(v+w))=\zeta^{-1}(\zeta(u) \zeta(v))+\zeta^{-1}(\zeta(u) \zeta(w)) .
$$

The general solution is $\zeta(u)=(A u)^{C}$ where $A$ and $C$ are constants.13 Substituting back into eq. (18) gives $P(u, v)=A u v$ or $\xi(a b)=A \xi(a) \xi(b)$. The constant $A$ can be absorbed into a new function $\psi(a)=A \xi(a)$, so that, finally, the and operation can be conveniently represented by a simple product rule,

$$
\psi(a b)=\psi(a) \psi(b)
$$

while the sum rule remains unaffected,

$$
\psi\left(a \vee a^{\prime}\right)=\psi(a)+\psi\left(a^{\prime}\right) .
$$

To summarize: A quantitative representation of the relations between setups can be obtained by assigning a number $\psi(a)$ to each setup $a$. Except for the crucial requirement of consistency there seems to exist a considerable arbitrariness in the actual choice of $\psi(a)$ but this is largely illusory: Any consistent assignment is equivalent (i.e., a mere change of variables) to an assignment where and and or are represented by the product and the sum rules. Complex numbers 
assigned in this particularly convenient way are called "amplitudes". This is our main result.

A first application of these ideas arises from the observation that a single filter that is totally covered with holes is equivalent to having no filter at all. Suppose, for simplicity, that the positions of our idealized particle lie on a discrete lattice, then, using eqs. (6) and (7), we have (choose $t$ so that $t_{i}<t<$ $\left.t_{f}\right)$

$$
\left[x_{f}, x_{i}\right]=\underset{\text { all } \vec{x} \text { at } t}{\vee}\left(\left[x_{f}, x_{t}\right]\left[x_{t}, x_{i}\right]\right) .
$$

Thus motion over a long distance can be analyzed in terms of motion over shorter steps. The fundamental quantum equation of motion is the equation for the corresponding amplitudes. Using the sum and product rules, eqs. (20) and (21), we get

$$
\psi\left(x_{f}, x_{i}\right)=\sum_{\text {all } \vec{x} \text { at } t} \psi\left(x_{f}, x\right) \psi\left(x, x_{i}\right) .
$$

To write it in Schrödinger form one introduces, 14 the notion of a state described by a wave function.

Consider the (not unusual) situation where all reference to the starting point $\left(\vec{x}_{i}, t_{i}\right)$, and to those interactions prior to time $t$ can be ignored. Then the amplitude $\psi\left(\vec{x}, t ; \vec{x}_{i}, t_{i}\right)$ can be simply written as $\Psi(\vec{x}, t)$, which we will call the wave function. Writing eq. (23) as

$$
\Psi\left(\vec{x}_{f}, t_{f}\right)=\sum_{\text {all } \vec{x} \text { at } t} \psi\left(\vec{x}_{f}, t_{f} ; \vec{x}, t\right) \Psi(\vec{x}, t),
$$

shows that $\Psi(\vec{x}, t)$ is sufficient to determine future evolution. The wave function $\Psi(\vec{x}, t)$ represents those features of the particle's history prior to $t$ that are relevant to its evolution after $t$; one might say that $\Psi(\vec{x}, t)$ represents the state of the particle at $t$, or perhaps better, that it represents the preparation procedure.

Differentiating eq. (24) with respect to $t_{f}$ and evaluating at $t_{f}=t$ gives the linear Schrödinger equation

$$
-i \hbar \frac{\partial \Psi\left(\vec{x}_{f}, t\right)}{\partial t}=\sum_{\text {all } \vec{x} \text { at } t} H\left(\vec{x}_{f}, \vec{x}, t\right) \Psi(\vec{x}, t),
$$

where $H$ is the derivative

$$
\left.\frac{\partial \psi\left(\vec{x}_{f}, t^{\prime} ; \vec{x}, t\right)}{\partial t^{\prime}}\right|_{t^{\prime}=t} \equiv \frac{i}{\hbar} H\left(\vec{x}_{f}, \vec{x}, t\right) .
$$

Once the assumption is made that the relations among setups are represented quantitatively in terms of amplitudes, consistency requires that the time evolution of quantum states be given by a necessarily linear Schrödinger equation. The question of whether non-linear forms of quantum mechanics are possible should not be addressed at the shallow level of seeking modified Hamiltonians 
but should be rephrased at a deeper level as a question about how relations among physical entities are to be represented by relations among mathematical ones.

We conclude with a few brief remarks. First, in the quantum logic approach linearity follows from the reasonable assumption that time evolution is induced by a symmetry transformation of the proposition system, 8 , 15 which, as a consequence of Wigner's theorem, is represented by operators that are linear and unitary. No such assumption is made here.

As introduced here amplitudes play the crucial role of providing a consistent representation of the relations between various idealized experimental setups but the important issue of how to use these amplitudes to predict the outcomes of experiments was not addressed. This gap is filled in a forthcoming paper 13 where we prove that the amplitudes must be interpreted according to Born's probability rule.

Finally, we emphasize that this approach yields the standard quantum mechanics with all its virtues and faults. Important questions such as why complex numbers instead of other mathematical objects with the required associativity

and distributivity, or those questions associated to the quantum mechanics of macroscopic objects are left open.

I am indebted to C. Rodriguez, A. Inomata, P. Zambianchi, and J. Kimball for valuable discussions and many insightful remarks.

\section{References}

[1] I. Bialynicki-Birula and J. Mycielski, Ann. Phys. (NY) 100, 62 (1976); A. Shimony, Phys. Rev. A20, 394 (1979); S. Weinberg, Phys. Rev. Lett. 62, 485 (1989), and Ann. Phys. (NY) 194, 336 (1989).

[2] C. G. Shull, et al., Phys. Rev. Lett. 44, 765 (1980); R. Gahler, A. G. Klein, and A. Zeilinger, Phys. Rev. A23, 1611 (1981); J. Bollinger et al., Phys. Rev. Lett. 63, 1031 (1989).

[3] N. Herbert, Found. Phys. 12, 1171 (1982); W. K. Wooters and W. Zurek, Nature 299, 802 (1982); N. Gisin, Helv. Phys. Acta 62, 363 (1989) and Phys. Lett. 143, 1 (1990); J. Polchinski, Phys. Rev. Lett. 66, 397 (1991); H. Scherer and P. Busch, Phys. Rev. 47, 1647 (1993).

[4] R. T. Cox, Am. J. Phys. 14, 1 (1946).

[5] E. T. Jaynes, "E. T. Jaynes: Papers on Probability, Statistics and Statistical Physics," edited by R. D. Rosenkrantz (Reidel, Dordrecht, 1983).

[6] R. P. Feynman, R. B. Leighton and M. Sands, "The Feynman Lectures on Physics," Vol. 3 (Addison-Wesley, 1965).

[7] D. Finkelstein, Trans. NY Acad. Sci. 25, 621 (1963). 
[8] J. M. Jauch, "Foundations of Quantum Mechanics" (Addison-Wesley, 1968).

[9] "The Logico-Algebraic Approach to Quantum Mechanics," C. A. Hooker (Ed.), Vols. I and II (Reidel, Dordrecht, 1979).

[10] See e.g., C. J. Isham, J. Math. Phys. 35, 2157 (1994) and R. B. Griffiths, Phys. Rev. A54, 2759 (1996) and references therein.

[11] S. Youssef, Mod. Phys. Lett. 6, 225 (1991); ibid. 9, 2571 (1994); Phys. Lett. A204, 181 (1995).

[12] Since the words 'consistency' and 'history' both appear in this letter the reader might be led to suspect a connection with 'consistent histories'. We hasten to point out that, beyond the shared subject of quantum theory, there is no connection. The 'consistent histories' approach seeks to clarify issues of interpretation by introducing rules (the consistency conditions) which restrict discourse to a subset of histories within which probabilities can be assigned. Our goal is to justify the formalism itself; we consider propositions to which amplitudes can be assigned.

[13] A. Caticha, Phys. Rev. A57, 1572 (1998).

[14] R. P. Feynman, Rev. Mod. Phys. 20, 267 (1948); R. P. Feynman and A. R. Hibbs, "Quantum Mechanics and Path Integrals," (McGraw-Hill, NY 1965).

[15] T. F. Jordan, Am. J. Phys. 59, 606 (1991); N. Gisin, Am. J. Phys. 61, 86 (1993). 